ISSN 1997-342X (Online), ISSN 1991-8631 (Print)

Original Paper http://ajol.info/index.php/ijbcs http://indexmedicus.afro.who.int

\title{
Gestion des forêts domaniales en période de conflits : cas de la forêt classée du Haut-Sassandra, Centre-Ouest de la Côte d'Ivoire
}

\author{
Akoua Tamia Madeleine KOUAKOU ${ }^{1 *}$, Yao Sadaiou Sabas BARIMA ${ }^{1}$, \\ Souleymane KONATE ${ }^{2}$, Issouf BAMBA ${ }^{1}$, Justin Yatty KOUADIO ${ }^{3}$ et Jan BOGAERT ${ }^{4}$ \\ ${ }^{1}$ Université Jean Lorougnon Guédé, Unité de Formation et de Recherche en Environnement, BP 150 Daloa, \\ Côte d'Ivoire. \\ ${ }^{2}$ Université Nangui Abrogoua, Unité de Formation et de Recherche en Sciences de la Nature, 02 BP 801 \\ Abidjan 02, Côte d'Ivoire. \\ ${ }^{3}$ Université Jean Lorougnon Guédé, Unité de Formation et de Recherche en Agroforesterie, BP 150 Daloa, \\ Côte d'Ivoire. \\ ${ }^{4}$ Université de Liège, Gembloux Agro-Bio Tech, Passage des Déportés 2, B-5030 Gembloux, Belgique. \\ *Auteur correspondant, E-mail : tamiakouakou01@gmail.com;Tel : +22547355441.
}

\section{REMERCIEMENTS}

Cette étude a été réalisée avec le soutien du Ministère de l'Enseignement Supérieur et de la Recherche Scientifique de Côte d'Ivoire dans le cadre de la mise en ouvre du Contrat de Désendettement et de Développement (C2D) avec l'appui technique de l'Institut de Recherche pour le Développement (IRD). Ce ministère a également octroyé une bourse de stage à Kouakou A pour l'analyse des données. L'étude a aussi bénéficié du soutien du programme GEOFORAFRI «renforcement des capacités et accès aux données satellitaires pour le suivi des forêts en Afrique " financé par le Fonds français pour l'Environnement Mondial et du Programme d'Appui Stratégique à la Recherche Scientifique en Côte d'Ivoire (PASRES), de l'Académie des Sciences, des Arts, des Cultures d'Afrique et des Diasporas africaines (ASCAD). L'accès à la Forêt Classée du Haut-Sassandra a été possible grâce à l'accord de la Société de Développement des Forêts en Côte d'Ivoire (SODEFOR).

\section{RESUME}

Des travaux antérieurs ont montré que les conflits armés des années 2000 en Côte d'Ivoire ont entrainé une forte anthropisation de la forêt classée du Haut-Sassandra située au Centre-Ouest du pays. L'objectif de la présente étude était de déterminer l'impact de la présence des différentes forces militaires sur la dynamique de cette forêt pendant la période des conflits. La méthodologie a consisté en la division de la forêt en trois zones, Nord, Centre et Sud, suivant les types de forces présentes pendant la période des conflits. La dynamique spatiotemporelle de ces zones a été déterminée à partir de la classification supervisée de trois images Landsat datant de 2002, 2006 et 2013. Une matrice de transition, un indice de stabilité et des indices de composition ont servi à analyser les changements intervenus dans les paysages pendant les périodes d'étude. La comparaison des zones à travers les indices indique qu'il n'y a pas de différence significative entre elles. Il apparaît ainsi que les trois zones subi la même dynamique de déforestation pendant la période de conflits. La présence des forces 
internationales dans les parties Centre et Sud de la forêt classée du Haut-Sassandra n'aurait pas empêché leur infiltration et leur déboisement.

(C) 2017 International Formulae Group. All rights reserved.

Mots clés : forces militaires, dynamique forestière, anthropisation, fragmentation.

\title{
Management of protected forest during the period of conflict: the case of the classified forest of Haut-Sassandra, Central West of Côte d'Ivoire
}

\begin{abstract}
Previous work has shown that armed conflicts of the 2000s in Côte d'Ivoire have led to a strong human impact on the classified forest of Haut-Sassandra located in the west-center of the country. The objective of this study was to determine the impact of the presence of the various military forces on the dynamics of the forest during the conflicts period. The methodology consisted of dividing the forest into three zones, North, Center and South, according to the types of forces present during the conflict period. The spatial and temporal dynamics of these areas was determined from the supervised classification of three Landsat images from 2002, 2006 and 2013. A transition matrix, a stability index, and compositional index were used to analyze changes in landscapes during study periods. The comparison of the areas through the index indicates that there is no significant difference between them. It appears that the three zones have suffered the same dynamics during the period of conflict. Therefore, the presence of international forces in parts of Central and South of the classified forest of Haut-Sassandra did not prevent their infiltration and deforestation.
\end{abstract}

(C) 2017 International Formulae Group. All rights reserved.

Keywords: Military forces, forest dynamics, anthropization, fragmentation.

\section{INTRODUCTION}

Le $\mathrm{XXI}^{\mathrm{e}}$ siècle est marqué par une dégradation croissante des paysages, notamment sous les tropiques (Ariori et Ozer, 2005 ; Filho et Steiner, 2005) conduisant à une réduction du couvert forestier naturel. Ce recul des massifs forestiers est plus préoccupant dans les pays en voie de développement dont ceux de l'Afrique de l'Ouest (N'da et al., 2008). Selon la FAO (2012), de 2000 à 2010 les surfaces forestières de l'Afrique de l'Ouest ont été réduites de $20 \%$. En Afrique comme partout dans le monde, les actions humaines, dont l'agriculture, sont à la base de cette situation (OFAC, 2012).

Par ailleurs, les conflits armés constituent l'une des causes importantes de l'effondrement des ressources naturelles comme ce fut le cas de plusieurs pays africains dont le Soudan et la République
Démocratique du Congo (PNUE, 2006; 2011).

En Côte d'Ivoire, les conflits armés qui ont duré une décennie, ont eu un impact considérable sur le milieu naturel en général et les espaces protégés en particulier (Chatelain, 2015; PNUE, 2015). Cette période a occasionné une importante déforestation due essentiellement à la conversion du couvert forestier des forêts classées en systèmes agricoles comme ce fut le cas de la forêt classée du Haut-Sassandra (FCHS) (Barima et al., 2016).

Avant les conflits, malgré l'essor de la culture du cacaoyer dans la région du Centre Ouest ivoirien, la FCHS fut épargnée de toute infiltration massive pour constituer l'une des forêts les mieux protégées de la Côte d'Ivoire (Oszwald, 2005). Plusieurs travaux ont été effectués sur l'impact des conflits sur la FCHS (Kouakou et al., 2015a, Sangne et al., 2015, 
Barima et al., 2016). Ces travaux tenaient compte pour certains de la partie Nord uniquement (Kouakou et al., 2015a, Sangne et al., 2015) et pour d'autres de la totalité de cet espace (Barima et al., 2016; Zanh et al., 2016). Tous ces travaux ont montré à travers l'analyse de la dynamique spatio-temporelle une anthropisation marquée par une fragmentation de la classe forestière. Ces auteurs ont noté un recul considérable du couvert forestier au profit des espaces cultivés ainsi qu'une perte de la diversité floristique. Ces travaux, bien qu'ayant apporté des informations importantes sur la dynamique de la forêt pendant la période de conflits, ne renseignent pas sur l'implication des forces militaires dans la gestion de cet espace domanial. Pourtant, ces informations pourraient être importantes pour la conservation des ressources naturelles pendant les périodes des conflits armés, en ce sens qu'elles pourraient aider dans les prises de décisions liées au règlement des conflits par la communauté internationale. En effet, en l'absence des autorités forestières, la FCHS était sous l'autorité des forces militaires, internationales et groupements militaires rebelles à l'autorité gouvernementale.

De la présence de ces différentes administrations découle l'hypothèse de cette étude qui stipule que la présence des forces internationales au Centre et au Sud de la FCHS pendant les conflits aurait permis une meilleure conservation de ces zones par rapport à la zone Nord sous administration des groupements rebelles avec une différence entre les dynamiques des zones Centre et Sud. En effet le Sud, du fait de sa proximité avec la zone gouvernementale serait sous influence de la population de cette zone mais aussi de celle de la zone de confiance. D'où une étude individuelle de chacune des zones, Nord, Centre et Sud. Ainsi l'objectif de cette étude est de déterminer les dynamiques des zones Nord, Centre et Sud de la FCHS pendant la période des conflits, afin de déterminer l'impact de la présence des différentes forces militaires sur ces dynamiques.

\section{MATERIEL ET METHODES}

Présentation de la zone d'étude

La zone d'étude est la forêt classée du Haut-Sassandra (FCHS) située dans la région du Haut-Sassandra au Centre-Ouest de la Côte d'Ivoire (Figure 1). Elle appartient au domaine forestier permanent de l'Etat ivoirien depuis le 23 novembre 1974. Elle est située entre $6^{\circ} 52^{\prime}$ et $7^{\circ} 24^{\prime}$ de latitude Nord et $6^{\circ} 59^{\prime}$ et $7^{\circ} 10^{\prime}$ de longitude Ouest. Elle s'étendait sur une superficie de 102400 ha à sa création. La FCHS appartenait de façon générale à la zone de forêt dense semi-décidue à Celtis spp. et Triplochiton scleroxylon et est caractérisée par une importante diversité floristique. Le sol de la FCHS, comme pour l'ensemble de la région $\mathrm{du}$ Haut-Sassandra est $\mathrm{du}$ type ferralitique qui est connu pour sa bonne aptitude agricole (Koffie-Bipko et Kra, 2013). De ce fait cette région constitue l'une des régions à forte production agricole aussi bien pour le vivrier que pour les cultures d'exportation.

Cette potentialité agricole est aussi favorisée par un climat tropical humide offrant une importante pluviométrie. La région du Haut-Sassandra est fortement peuplée avec plus des deux tiers de la population en milieu rural.

\section{Cartographie de l'occupation du sol}

La détermination des changements dans l'occupation du sol de la FCHS dans ses zones Nord, Centre et Sud pendant la décennie de conflits a nécessité l'utilisation de trois images satellites issues du capteur Landsat des années 2002, 2006 et 2013. Les deux premières images datent respectivement du 13 décembre 2002 et du 08 décembre 2006 et sont issues du capteur ETM+. La troisième image date du 19 décembre 2013 et provient $\mathrm{du}$ capteur OLI TIRS. Toutes ces images de $30 \mathrm{~m}$ de résolution chacune, ont été acquises pendant la saison sèche. En effet, les images acquises pendant cette saison présentent une grande différence spectrale entre les classes d'occupation du sol et permettent de différencier les espaces anthropisés (culture, 
jachère) des espaces de végétation naturel (forêt) (Barima et al., 2009; Oszwald et al., 2010). Aussi au cours de la saison sèche les effets atmosphériques sur les images sont réduits (Chander et Markham, 2003).

Avec le logiciel ENVI 4.3, le traitement de ces images a commencé par la correction géométrique à partir de point de repère au sol suivi d'une correction atmosphérique.

Les images ont subi une classification supervisée à partir de l'algorithme du maximum de vraisemblance sur une combinaison de trois bandes (proche infrarouge, rouge et vert). Pour ce faire, une composition colorée fausse couleur a été faite sur chacune des images (Figure 1) avec les trois bandes pour une meilleure distinction des classes d'occupation du sol, en mettant les bandes du proche infra-rouge dans le rouge, celles du rouge dans le vert et celles du vert dans le bleue. Ces bandes sont respectivement les bandes $4(0,75-0,90 \mu \mathrm{m}), 3(0,63-0,69$ $\mu \mathrm{m})$ et $2(0,525-0,605 \mu \mathrm{m})$ pour les images de $2002(\mathrm{ETM}+)$ et 2006 et les bandes 5 $(0,845-0,885 \mu \mathrm{m}), 4(0,630-0,680 \mu \mathrm{m})$ et 3 $(0,525-0,600 \mu \mathrm{m})$ pour l'image de 2013. Plus la couleur est rouge, plus la végétation est dense. Les couleurs tendant vers le blanc traduisent l'absence de végétation et la présence de sol et la présence de l'eau est marquée par la couleur bleu (Figure 1). Les classes d'occupation du sol retenues pour cette étude sur la base des connaissances de terrain sont la classe «Forêt», la classe «Forêt dégradée - culture », la classe « Sol nu - habitat» et la classe «Eau ». Cependant, la classe eau n'a pas été prise en compte dans le rendu cartographique car constituée essentiellement du fleuve Sassandra à la limite Ouest de la forêt.

Pour la classification, 15 zones d'entrainement pour chacune des classes ont été délimitées sur les compositions colorées à partir de données de terrain.

Pour la vérification de la qualité des classifications, une matrice de confusion et l'indice de Kappa ont été générés pour chacune des images à partir de 360 zones de contrôle délimitées sur le terrain.

\section{Analyse du niveau d'anthropisation des zones Nord, Centre et Sud de la forêt classée du Haut-Sassandra}

Pour une analyse de la dynamique du paysage des trois zones de la FCHS, les images classifiées ont été divisées en trois parties correspondant au Nord, au Centre et au Sud de la FCHS couvrant chacune une superficie d'environ $21 \mathrm{~km} \times 19 \mathrm{~km}$ (Figure 1). Cette subdivision est faite sur la base des limites administratives correspondant à la partie Nord sous contrôle des groupements rebelles à l'autorité gouvernementale et la zone dite de «confiance» ou zone tampon définie et contrôlée par l'ONU. Cette zone de confiance a été divisée en deux parties de mêmes dimensions, pour déterminer une éventuelle influence des populations de la zone gouvernementale sur la partie méridionale de la FCHS.

La proportion d'occupation des classes (PC), le nombre de taches (NT), l'aire moyenne des taches (AIRE $E_{\mathrm{MOY}}$ ) et l'indice de dominance de la plus grande tache (LPI) ont été calculées. La proportion d'occupation des classes (PC) mesure la proportion du paysage occupée par les différents types d'occupation $\mathrm{du}$ sol. Cet indice permet de déterminer le type d'occupation du sol dominant le paysage. Le nombre de taches associé à l'aire moyenne des taches permet d'évaluer le niveau de fragmentation. L'indice de dominance (LPI) est le rapport de l'aire de la plus grande tache de la classe et l'aire totale de la classe (McGarigal, et Cushman, 2002). Cet indice qui prend des valeurs entre 0 et 100 , permet également de déterminer le niveau de fragmentation de la classe. Plus la valeur de la dominance de la plus grande tache tend vers 100 moins la classe est fragmentée et lorsque cette valeur tend vers 0 la classe est fragmentée (Bogaert et al., 2002). D'une façon générale, l'évolution des différents indices permet de déterminer le processus de transformation dominant le paysage (Bogaert et al., 2004 ; Barima et al., 2009). Nous avons 
également appréhendé le niveau de l'anthropisation des trois zones en composant une matrice de transition et en calculant un indice de stabilité pour déterminer les conversions entre les différentes classes d'occupation du sol. L'indice de stabilité est le rapport de la somme des valeurs de la diagonale et de la somme des valeurs hors diagonal de la matrice de transition (Bogaert et al., 2014).

Pour tenir compte de l'ensemble de l'hétérogénéité de chaque zone et pour avoir une série de répétition des données, nous avons subdivisé chacune des zones en huit secteurs de 2000 ha. Ces secteurs étaient disposés de façon aléatoire dans chacune des zones et au même emplacement pour chacune des années (Figure 2). Ainsi nous avions pour chaque année huit secteurs pour chacune des zones, soit 72 secteurs pour l'ensemble des zones sur la période de l'étude. Les différents indices de paysages utilisés pour l'analyse de variance ont été calculés pour chaque classe de chaque secteur pour les trois zones et pour chacune des années. Cette opération a permis d'obtenir une série de données pour l'analyse de variance.

\section{Analyse statistique}

Pour comparer les dynamiques des zones Nord, Centre et Sud, des tests d'analyse de variance de la moyenne (ANOVA) ont été effectués sur les indices mentionnés ci-dessus (PC, NT, AIRE $\mathrm{MOY}_{\mathrm{MO}}$ et LPI) à l'aide du logiciel R. Ces analyses de variance à partir de l'ANOVA ont été faites après vérification des conditions d'application. Lorsqu'une différence significative est observée entre les trois zones après l'analyse de variance, le test de comparaison multiple de Tukey a été effectué pour déterminer les zones homogènes.

Les données qui ont servi à ces comparaisons ont été obtenues dans des secteurs.

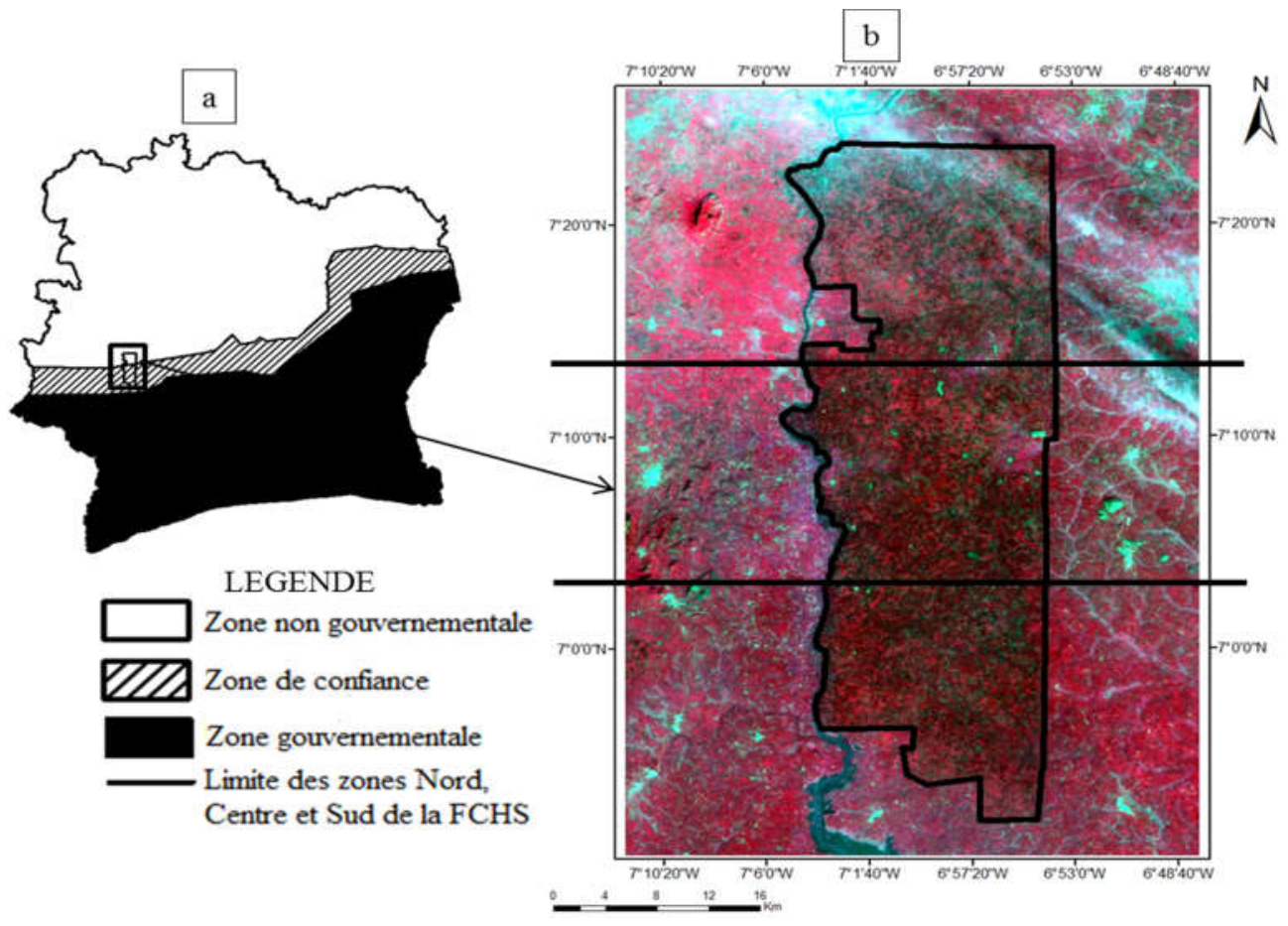

Figure 1 : Situation de la Forêt classée du Haut-Sassandra en Côte d'Ivoire (a) et limites des découpes (Nord, Centre et Sud) sur une composition fausse couleur de l'image de 2013 (b) à partir des bandes $5(0,845-0,885 \mu \mathrm{m}), 4(0,630-0,680 \mu \mathrm{m})$ et $3(0,525-0,600 \mu \mathrm{m})$ du capteur OLI TIRS. 

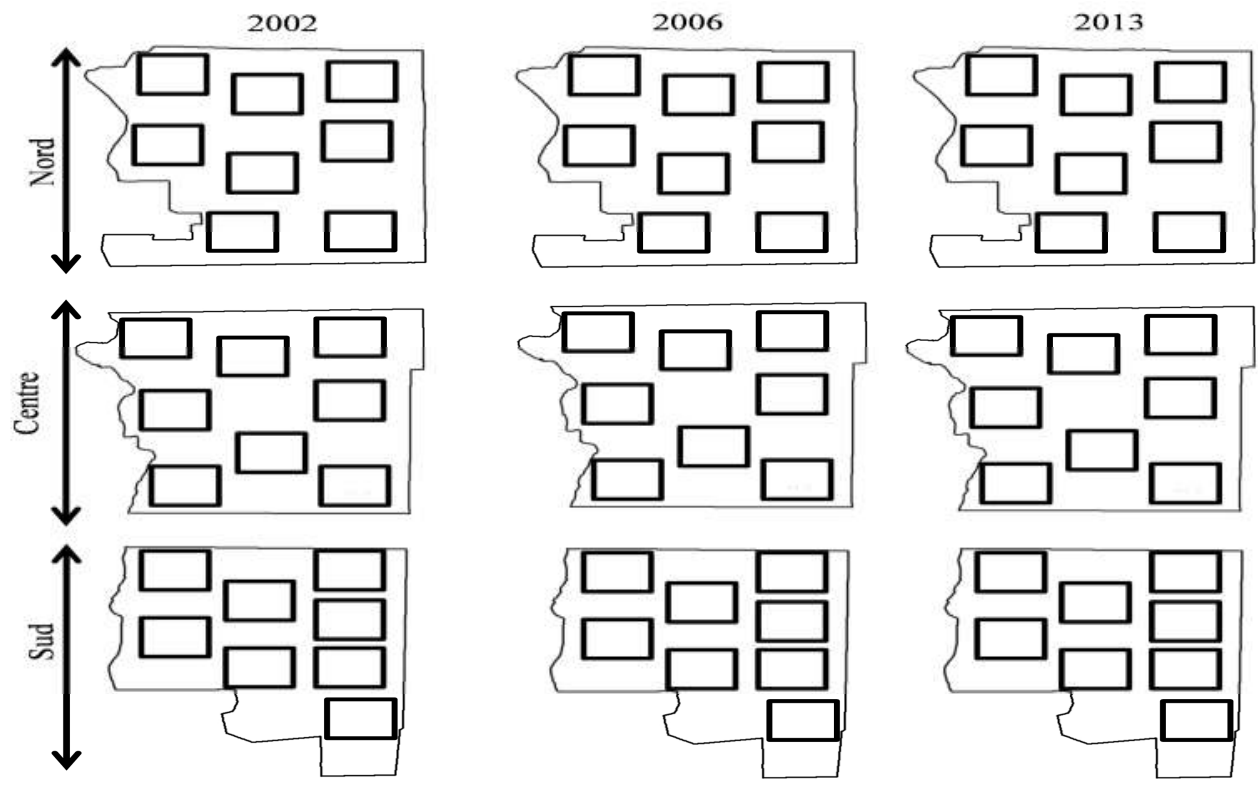

Figure 2 : Représentation des différents secteurs ayant servi aux comparaisons des zones Nord, Centre et Sud de la forêt classée du Haut-Sassandra des années 2002, 2006 et 2013.

\section{RESULTATS \\ Cartographie et vérification des classifications}

La Figure 3 presente les cartes d'occupation $\mathrm{du}$ sol obtenues après classification supervisée des images Landsat de la FCHS de 2002, 2006 et 2013. Elles présentent la repartition spatiale de chacune des classes d'occupation du sol.

L'évaluation de la qualité des classifications a donné une précision globale élévée pour chacune des images avec une valeur de $97,99 \%$ pour l'image de 2002 , $95,87 \%$ pour celle de 2006 et 96,79 pour celle de 2013. Ces precisions sont confirmées par le coefficient de Kappa qui est de 0,97 en 2002, 0,94 en 2006 et 0,95 en 2013 (Tableau 1). Pour chacune de ces classifications, on note une bonne distinction entre les classes avec cependant quelques confusions dont les plus importantes se trouvent entre les classes "Forêt» et «Forêt dégradée - culture ». L'image de 2002 présente la meilleure classification de la classe «Forêt» avec $99,4 \%$ des pixels bien rangés et seulement
$0,7 \%$ rangé en «Forêt dégradé - culture ». L'image de 2006 a la plus grande confusion entre ces deux classes avec $4,1 \%$ des pixels de la classe «Forêt» rangés en « Forêt dégradée - culture » contre 2,5\% pour l'image de 2013 (Tableau 1).

\section{Dynamique des zones Nord, Centre et Sud} de la forêt classée du Haut - Sassandra

Les résultats montrent la forte croissance des classes «Forêt dégradée culture » et «Sol nu - habitat» au détriment de la classe «Forêt» dans chacune des zones. $\mathrm{Au}$ Nord, en 2002 le paysage était constitué presque dans sa totalité de la classe forestière representée par une seule tache avec une superficie de 34390 ha. Cette classe a subi une augmentation du nombre et une diminution de l'aire moyenne des taches pour occuper à la fin des conflits seulement un tiers du paysage (Tableau 2). Au Centre, le nombre de taches forestières connait également une importante croissance passant de 3 en 2002 à 37 en 2006 et 791 en 2013 avec une diminution de l'aire moyenne et donc de la proportion 
d'occupation du sol (Tableau 2). Ainsi, l'aire moyenne des taches forestières chute et est 500 fois moins importante en 2013 qu'en 2002 (Tableau 2). Comme au Nord et au Centre, la classe "Forêt» a connu une fragmentation au Sud traduite par une augmentation du nombre de taches suivi d'une diminution de leur aire moyenne (Tableau 2). Dans cette dernière zone le nombre de taches est passé de 49 en 2002 à 113 en 2006 et à 306 en 2013 soit une augmentation de 524\% de 2002 à 2013.

La période de conflits fut une période de forte conversion des surfaces forestières des zones Nord, Centre et Sud de la FCHS. Cette conversion est également indiquée par la baisse de façon générale de l'indice de dominance de la plus grande tache au niveau de la classe «Forêt» et son augmentation dans les autres classes entre 2002 et 2013 (Tableau 2). Cependant, le Nord semble le plus anthropisé avec le plus fort taux de régression de l'aire moyenne des taches forestières et une augmentation importante $\mathrm{du}$ nombre des taches forestières.

Entre 2002 et 2006 la dynamique de la classe forestière dans les trois zones a été marquée par une perte de superficie au profit des autres classes. Cependant, en 2006 chacune des zones disposait encore d'une importante superficie forestière (Tableau 3) . Au Nord, presque la totalité des superficies du paysage occupée par la «Forêt» en 2002 n'a pas subit de transformation. Seulement 2,1\% et $0,5 \%$ de ces superficies ont été converties respectivement en «Forêt dégradée culture » et «Sol nu - habitat ». Au Centre, la conversion des forêts a été plus orientée vers la classe «Forêt dégradée - culture » avec cependant une conservation d'une proportion importante des forêts existant en 2002. Au cours de cette période, marquant la première moitié de la période des conflits le Sud fut la plus affectée par les activités anthropiques (Tableau 3). La seconde moitié de la période des conflits $(2006$ - 2013) est caracterisée par une chute importante des superficies forestières dans chacune des zones par rapport à la période 2002 - 2006 avec une accentuation au Nord caracterisée par un maintien de moins de la moitié des superficices du paysage occupées par les forêts en 2006. Au cours de cette période le Nord est marqué par une proliferation des sols nus et habitats. pourtant en 2006 on note seulemment $0,5 \%$ des superficies forestières de 2002 converties en « Sol nu - habitat», en 2013 la dynamique de la forêt indique une conversion du tiers des superficies du paysage occupées par la forêt en 2006 en «Sol nu habitat» (Tableau 3).

En resumé la période 2006 - 2013 fut celle des importantes pertes de couverture forestière dans chacune des zones. De 2002 à 2006 les actions anthropiques étaient plus marquées par la mise en place des cultures. De 2006 à 2013, en plus de la classe "Forêt dégradée culture », la classe «Sol nu - habitat» a connu une importante extension.

Les résultats montrent ainsi que la période 2006 - 2013 a été la plus importante dans le processus de transformation du paysage des zones Nord, Centre et Sud de la FCHS.

Au cours de la période des conflits, les différentes classes dans chacune des zones ont connu les mêmes processus de transformation. La classe forêstière a subi une augmentation du nombre de taches accompagnée d'une baisse de l'aire moyenne et de la dominance de la plus grande tache traduisant ainsi une fragmentation de celle-ci. Contrairement à la classe forestière, les classes «Forêt degradée - culture » et «Sol nu - habitat»ont été dominées par le processus de creation avec une augmentation du nombre de taches ainsi que de l'aire moyenne de celles-ci. Cette tendance à la creation de ces classes connait cependant une certaine variation exprimée par l'indice de dominance. De 2002 à 2006, cet indice connait une augmentation au niveau de ces deux classes dans chacune des zones. De 2006 à 2013, Au Nord, l'indice de dominance a subi une diminution dans la classe «Forêt degradée - culture » et une augmentation au niveau de la classe «Sol nu - habitat» suggerant ainsi une creation suivi d'une agrégation des taches dans cette dernière 
classe et une creation plus importante de taches isolées dans la première classe. $\mathrm{Au}$ centre et au Sud, le processus d'agrégation s'est plutôt observé au niveau de la classe «Forêt degradée - culture ».

Analyse comparative des zones Nord, Centre et Sud de la Forêt classée du HautSassandra

La méthodologie a donné lieu à une étude comparative des différentes zones permettant de mettre en évidence les différences suivant l'évolution des indices utilisés. Le Tableau 4 montre les valeurs de l'indice de stabilité de chacune des zones pour les différentes périodes de l'étude. Cet indice qui prend des valeurs élevées pour des paysages qui ont subi de faible dynamique, est pour la période $2002-2006,3$ fois plus élevé pour la zone Nord que les zones Centre et Sud. Au cours de cette période, la valeur de l'indice de stabilité dans la zone Nord indique que les valeurs sur la diagonale de la matrice de transition qui expriment le pourcentage de stabilité des classes, sont 30 fois plus élevées que celles hors diagonale. Quant aux zones Centre et Sud, les valeurs sur la diagonale sont respectivement 8 fois et 7 fois plus élevées que les valeurs hors diagonale. Le changement de l'indice de stabilité d'une zone à une autre semble avoir eu lieu uniquement que sur cette période. En effet, pour la seconde période (2006 - 2013) et pour l'ensemble de la période d'étude cet indice prend de faible valeur et connait une faible variation entre les zones (Tableau 4).

Les différences entre les zones en fonction des indices de composition ne suivent pas toujours le même ordre de significativité. En tenant compte de la proportion d'occupation du sol, les différences entre les zones ne sont pas significatives $(p>0,05)$ d'une année à une autre. Ce constat est le même pour l'aire moyenne des taches $(p>0,05)$ et la dominance de la plus grande tache $(p>0,05)$. Pour ce qui est du nombre de taches, il existe une différence significative $(p<0,01)$ entre les zones. Le Nord diffère significativement du Centre $(p<0,01)$ et du Sud $(p<0,01)$. Tandis qu'il n'y a pas de différence entre le Centre et le Sud $(p>0,05)$. D'une façon générale les trois zones ont subi la même évolution si l'on met à part le nombre de taches qui semble suivre une dynamique différente. Cet indice ne permettant pas à lui seul de mettre en évidence une différence entre les niveaux de fragmentation de la classe forestière dans les différentes zones, nous pouvons dire que la fragmentation dans les trois zones est du même ordre puisque de façon générale on note une augmentation et une diminution de l'aire moyenne des taches forestières. Quel que soit l'indice considéré il n'y a pas de différence entre le Centre et le Sud montrant que le Sud n'a pas subi une pression plus importante que celle du Centre.

Tableau 1 : Matrice de confusion et indice de Kappa issus de la vérification des classifications des images Landsat de 2002, 2006 et 2013 de la forêt classée du Haut-Sassadra.

\begin{tabular}{llcccc} 
& Forêt & $\begin{array}{c}\text { Forêt dégradée - } \\
\text { culture }\end{array}$ & $\begin{array}{c}\text { Sol nu - } \\
\text { habitat }\end{array}$ & Eau \\
\cline { 2 - 5 } $\mathbf{2 0 0 2}$ & 99,4 & 0,7 & 0,8 & 1,5 \\
Forêt dégradée- & 0,5 & 97,9 & 2,0 & 0,0 \\
culture & Sol nu-habitat & 0,1 & 1,4 & 97,2 & 0,5 \\
Eau & 0,0 & 0,0 & 0,0 & 98,0 \\
& Précision globale : $97,99 \%$ & & \\
\hline
\end{tabular}


A. T. M. KOUAKOU et al. / Int. J. Biol. Chem. Sci. 11(1): 333-349, 2017

\begin{tabular}{|c|c|c|c|c|c|}
\hline \multirow{6}{*}{2006} & Forêt & 97,3 & 4,1 & 1,6 & 1,3 \\
\hline & Forêt dégradé-culture & 2,5 & 94,3 & 1,6 & 2,0 \\
\hline & Sol nu-habitat & 0,2 & 0,8 & 96,6 & 0,6 \\
\hline & Eau & 0,0 & 0,8 & 0,2 & 96,1 \\
\hline & & Précision & : 95,87\% & & \\
\hline & & Coefficien & ppa : 0,94 & & \\
\hline \multirow{6}{*}{2013} & Forêt & 97,5 & 2,5 & 0,5 & 2,0 \\
\hline & Forêt dégradée-culture & 2,0 & 94,2 & 1,3 & 0,0 \\
\hline & Sol nu-habitat & 0,5 & 3,3 & 98,2 & 0,0 \\
\hline & Eau & 0,0 & 0,0 & 0,00 & 98,0 \\
\hline & \multicolumn{5}{|c|}{ Précision globale : 96,79\% } \\
\hline & \multicolumn{5}{|c|}{ Coefficient de Kappa : 0,95 } \\
\hline
\end{tabular}

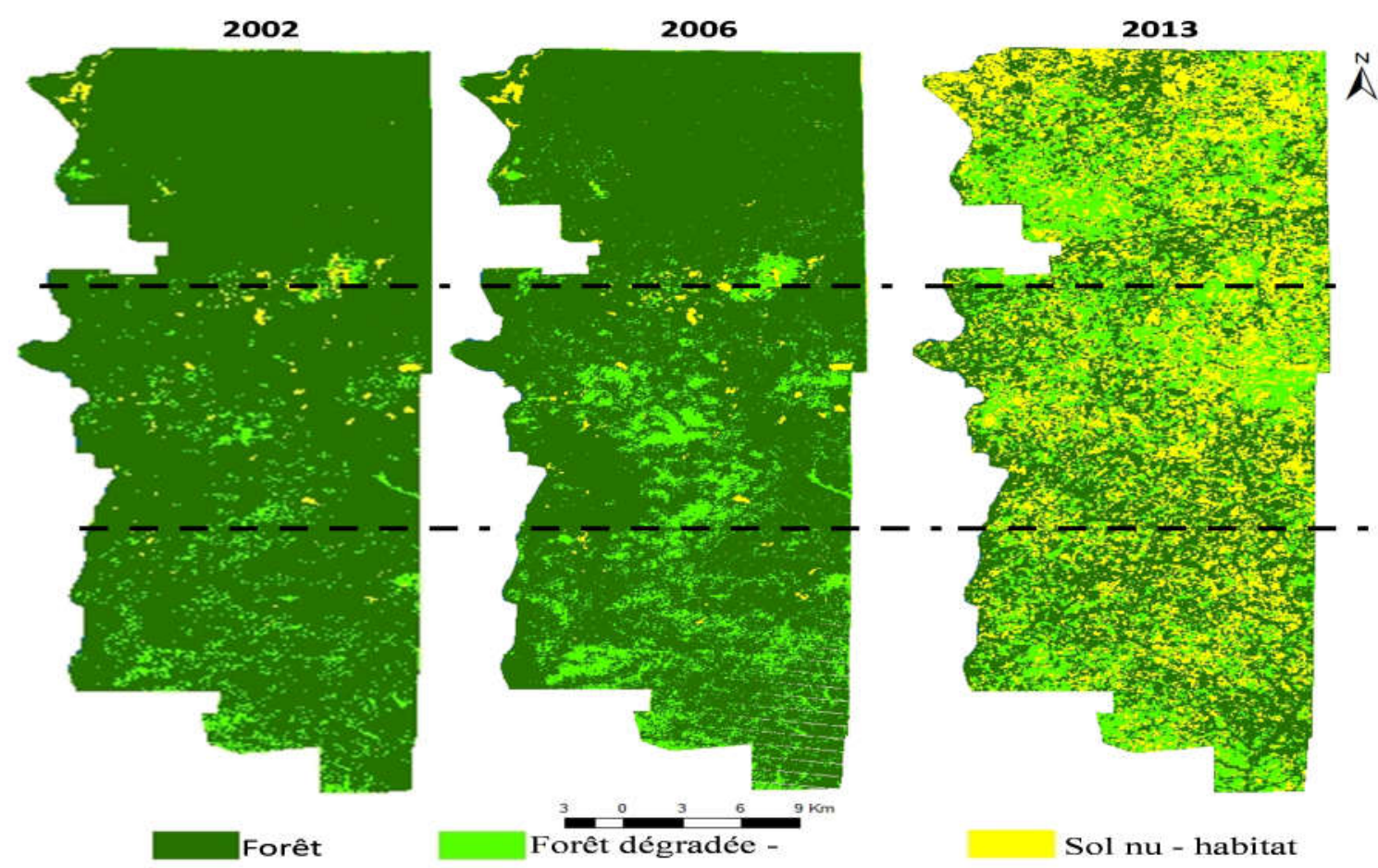

Figure 3 : Cartes d'occupation du sol de la forêt classée du Haut-Sassandra (Centre Ouest de la Côte d'Ivoire) en 2002, 2006 et 2013 et limites de ses zones Nord, Centre et Sud. 
Tableau 2 : Proportion d'occupation du sol (PC), nombre de taches (NT), l'aire moyenne des taches $\left(\mathrm{AIRE}_{\mathrm{MOY}}\right)$, l'indice de dominance de la plus grande tache (LPI) des classes d'occupation du sol de la forêt classée du Haut-Sassandra.
PC (\%)
NT
$\operatorname{AIRE}_{\text {MOY (ha) }}$
LPI (\%)

$\begin{array}{llllllllllll}2002 & 2006 & 2013 & 2002 & 2006 & 2013 & 2002 & 2006 & 2013 & 2002 & 2006 & 2013\end{array}$

\begin{tabular}{|c|c|c|c|c|c|c|c|c|c|c|c|c|c|}
\hline \multirow{3}{*}{ NORD } & \multirow{3}{*}{$\mathbf{F}$} & & & & \multirow[b]{2}{*}{1} & \multirow[b]{2}{*}{5} & \multirow{2}{*}{1333} & \multirow{2}{*}{34390,0} & \multirow{2}{*}{6712,0} & \multirow[b]{2}{*}{9,3} & \multirow{2}{*}{100,0} & \multirow{2}{*}{100,0} & \multirow{2}{*}{27,3} \\
\hline & & & & & & & & & & & & & \\
\hline & & 96,9 & 94,6 & 34,9 & & & & & & & & & \\
\hline & FD- & & & & 239 & 821 & 1446 & 2,1 & 1,6 & 7,3 & 21,1 & 44,8 & 31,7 \\
\hline & C & 1,5 & 3,7 & 29,6 & & & & & & & & & \\
\hline & SN- & & & & 209 & 260 & 1687 & 2,8 & 2,3 & 7,5 & 16,0 & 15,2 & 18,8 \\
\hline & H & 1,6 & 1,7 & 35,5 & & & & & & & & & \\
\hline
\end{tabular}

\begin{tabular}{lllllllllllllll}
\hline CENTRE & F & & & & 3 & 37 & 791 & 10394,0 & 752,5 & 18,1 & 100,0 & 99,9 & 56,3 \\
& & 94,0 & 83,9 & 43,3 & & & & & & & & & \\
& & & & & & & & & & & & & \\
& FD- & & & & 1160 & 1802 & 1872 & 1,4 & 2,7 & 4,5 & 9,6 & 10,2 & 20,3 \\
& & 4,7 & 14,7 & 25,6 & & & & & & & & & \\
& & & & & & & & & & & & & \\
& HN- & & & & 140 & 172 & 1801 & 2,9 & 2,6 & 5,7 & 14,9 & 13,3 & 7,6 \\
&
\end{tabular}

\begin{tabular}{|c|c|c|c|c|c|c|c|c|c|c|c|c|c|}
\hline \multirow[t]{4}{*}{ SUD } & \multirow[t]{2}{*}{$\mathbf{F}$} & & & & 46 & 113 & 306 & 555,4 & 200,6 & 48,9 & 99,8 & 99,1 & 91,8 \\
\hline & & 89,2 & 79,8 & 52,3 & & & & & & & & & \\
\hline & $\begin{array}{l}\text { FD- } \\
\text { C }\end{array}$ & 10,5 & 19,8 & 24,1 & 1973 & 2269 & 1686 & 1,5 & 2,5 & 4,1 & 20,5 & 11,7 & 20,1 \\
\hline & $\begin{array}{l}\text { SN- } \\
\text { H }\end{array}$ & 0,3 & 0,4 & 23,6 & 57 & 91 & 1907 & 1,5 & 1,4 & 3,6 & 17,7 & 14,7 & 3,3 \\
\hline
\end{tabular}

$\mathrm{F}=$ « Forêt », FD-C = « Forêt degradée - culture » et SN-H = « Sol nu - habitat ». Indices calculés sur base de classification d'images Landsat de 2002, 2006 et 2013. 
Tableau 3 : Matrice de transition des taux (\%) de conversion entre les classes d'occupation du sol de la forêt classée du Haut-sassandra des périodes 2002-2006 et 2006-2013.

\begin{tabular}{|c|c|c|c|c|c|c|c|c|c|c|c|c|c|}
\hline \multirow[b]{2}{*}{ 2002-2006 } & & \multicolumn{4}{|c|}{ Nord } & \multicolumn{4}{|c|}{ Centre } & \multicolumn{4}{|l|}{ Sud } \\
\hline & & $\mathbf{F}$ & $\begin{array}{l}\text { FD- } \\
\text { C }\end{array}$ & SN-H & TG & $\mathbf{F}$ & $\begin{array}{l}\text { FD- } \\
\text { C }\end{array}$ & SN-H & TG & $\mathbf{F}$ & $\begin{array}{l}\text { FD- } \\
\text { C }\end{array}$ & SN-H & TG \\
\hline & $\mathbf{F}$ & 94,3 & 2,1 & 0,5 & 96,9 & 83,6 & 10,2 & 0,2 & 94,0 & 78,7 & 10,4 & 0,2 & 89,3 \\
\hline & FD-C & 0,1 & 1,2 & 0,1 & 1,4 & 0,3 & 4,4 & 0,0 & 4,7 & 1,2 & 9,1 & 0,1 & 10,4 \\
\hline & SN-H & 0,1 & 0,4 & 1,2 & 1,7 & 0,1 & 0,1 & 1,1 & 1,3 & 0,0 & 0,0 & 0,3 & 0,3 \\
\hline & TG & 94,5 & 3,7 & 1,8 & 100,0 & 84,0 & 14,7 & 1,3 & 100,0 & 79,9 & 19,5 & 0,6 & 100,0 \\
\hline \multirow[t]{4}{*}{ 2006-2013 } & $\mathbf{F}$ & 34,2 & 26,9 & 33,4 & 94,5 & 38,4 & 19,9 & 25,7 & 84,0 & 44,4 & 16,6 & 19,0 & 80,0 \\
\hline & FD-C & 0,5 & 2,4 & 0,9 & 3,8 & 4,8 & 5,5 & 4,3 & 14,6 & 7,5 & 7,7 & 4,3 & 19,5 \\
\hline & SN-H & 0,2 & 0,3 & 1,2 & 1,7 & 0,1 & 0,1 & 1,2 & 1,4 & 0,1 & 0,1 & 0,3 & 0,5 \\
\hline & TG & 34,9 & 29,6 & 35,5 & 100,0 & 43,3 & 25,5 & 31,2 & 100,0 & 52,0 & 24,4 & 23,6 & 100,0 \\
\hline \multirow[t]{4}{*}{$2002-2013$} & $\mathbf{F}$ & 34,7 & 28,2 & 34,0 & 96,9 & 42,0 & 23,3 & 28,8 & 94,1 & 48,8 & 18,8 & 21,6 & 89,2 \\
\hline & FD-C & 0,1 & 1,0 & 0,3 & 1,4 & 1,2 & 2,3 & 1,2 & 4,7 & 3,5 & 5,2 & 1,9 & 10,6 \\
\hline & SN-H & 0,1 & 0,4 & 1,2 & 1,7 & 0,0 & 0,1 & 1,1 & 1,2 & 0,0 & 0,0 & 0,2 & 0,2 \\
\hline & TG & 34,9 & 29,6 & 35,5 & 100,0 & 43,2 & 25,7 & 31,1 & 100,0 & 52,3 & 24,0 & 23,7 & 100,0 \\
\hline
\end{tabular}

$\mathrm{F}=$ « Forêt », FD-C = « Forêt degradée - culture » et SN-H = « Sol nu - habitat », TG = total general. Matrice composée à partir de la classification d'images Landsat de 2002, 2006 et 2013.

Tableau 4 : Indice de stabilité de la forêt classée du Haut-Sassandra au cours de la période des conflits armés.

\begin{tabular}{llll}
\hline & Nord & Centre & Sud \\
\hline $\mathbf{2 0 0 2 - 2 0 0 6}$ & 30,3 & 8,1 & 7,4 \\
\hline $\mathbf{2 0 0 6 - 2 0 1 3}$ & 0,6 & 0,8 & 1,1 \\
\hline
\end{tabular}




\section{DISCUSSION}

\section{Gestion de la forêt classée du Haut- Sassandra de 2002 à 2013}

Les résultats de cette étude ont mis en évidence la régression des surfaces forestières dans la forêt classée du Haut-Sassandra pendant la période de conflits au profit des classes «Forêt dégradée - culture » et «Sol nu - habitat». Les transformations des couverts forestiers dans cet espace protégé sont essentiellement dues à la mise en place des cultures dominées par les cacaoyers (Assalé et al., 2016 ; Barima et al., 2016). Les cultures sont ainsi les principales causes du recul du couvert forestier en Afrique comme l'ont souligné Tente et al. (2011) en ce qui concerne le Bénin. Cette activité est aussi accompagnée de la prolifération des zones d'habitation dans la FCHS qui s'expliquerait par la volonté des populations de vivre à proximité de leur exploitation afin de faciliter leur entretien et de protéger leur récolte contre d'éventuels vols. Plusieurs études ont abordé l'infiltration de la FCHS par les agriculteurs avant et pendant la période des conflits (Oszwald, 2005; Kouamé, 1998). Ces infiltrations qui étaient relativement faibles se faisaient dans les zones Centre et Sud et étaient situées soit au cœur de la forêt, pour échapper aux agents de surveillance qui menaient régulièrement des opérations de déguerpissement pour freiner la dégradation, soit autour du site d'implantation de la société chargé d'aménager la FCHS. Aujourd'hui, les plantations au sein de la FCHS sont constatées sur toute l'étendue de sa surface. Cette généralisation des implantations agricoles s'est réalisée à partir de la période de conflits.

Les missions et enquêtes de terrain ont permis de montrer que l'essentiel de la population infiltrée dans la FCHS provient principalement de pays voisins à la Côte d'Ivoire confirmant ainsi les résultats de Kouakou et al. (2015a) et Assalé et al. (2016).
Ces migrations seraient dues aux conditions défavorables à l'agriculture dans les pays d'origine tel que le Burkina-Faso (Dibloni et al., 2009). Les populations migrantes auraient été dirigées vers la FCHS du fait de la saturation foncière dans les villages jouxtant cette zone protégée, rendant alors l'accès à la terre difficile pour les migrants. Par ailleurs, certaines populations autochtones auraient dirigé les migrants vers la FCHS par crainte de se voir exproprier le peu de terre qui leur reste par les migrants.

La fragmentation de la classe forestière de la FCHS s'est accompagnée de la perte de biodiversité. Les travaux de Kouakou et al. (2015a; 2015b) ont montré une perte de diversité floristique dans la partie Nord de la forêt pendant la période de conflits. Cette forêt qui était riche de plusieurs espèces floristiques contribuant à plus de $25 \%$ à la diversité floristique générale de la Côte d'Ivoire (Kouamé, 1998) a perdu dans sa partie Nord pendant la période de conflits plus de $12 \%$ de sa diversité floristique.

La fragmentation impacte la diversité aussi bien animale que végétale (Dibloni et al., 2009) à travers la réduction de la taille des fragments d'habitat naturel qui conduit à une diminution de la taille des populations. Ainsi la fragmentation affecte la biodiversité à travers l'isolement des fragments qui entraine une limitation du déplacement des espèces, leur présence, leur abondance et leur diversité (Debinski et al., 2001, Arroyo-Rodriguez et al., 2009 ) suivant cependant les caractéristiques de chacune d'entre elles. Les fortes pressions anthropiques (agriculture, exploitation forestière) que subit la FCHS menacent ainsi le maintien de la biodiversité primaire au profit des espèces secondaires à travers l'isolement des fragments forestier (Bakayoko et al., 2011; Martin, 2008). L'anthropisation, est ainsi responsable à moyen et long terme, de la baisse de diversité spécifique (Dan et al., 2012; Bamba, 2010; Barima et al., 2010).

Si en Côte d'Ivoire, la dégradation du couvert forestier a été induite par des infiltrations pour l'agriculture, ailleurs en Afrique les pressions sur les ressources naturelles pendant les conflits armés sont 
généralement dues aux poids des réfugiés (Havyarimana, 2015) et à l'exploitation illicite des ressources forestières (Hansen et al., 2008).

En somme, les perturbations d'origine anthropique modifient la dynamique des paysages et les conflits armés constituent une cause incontestée de ces modifications.

Si l'impact des conflits sur la dégradation des milieux naturels est indéniable, le rôle des différents types de forces militaires dans la conservation de ces milieux est aussi important à analyser. C'est à cette échelle d'observation que nous avons porté nos analyses. Pour les trois zones étudiées la période des conflits a entrainé une régression des surfaces forestières quel que soit les forces en présence (Barima et al., 2016). De 2002 à 2006, les changements de la couverture forestière ne furent pas importants dans les trois zones, par ailleurs, le degré d'anthropisation dans la zone Nord est encore plus réduit que dans les deux autres zones. Le Nord de la FCHS situé dans la zone sous contrôle de groupements rebelles semble avoir bénéficié d'une meilleure surveillance par rapport au Centre et au Sud sous administration des forces internationales. Les forces internationales déployées en cas de conflits armés ont pour mission principale de contrôler la mise en application du cessez-lefeu. Secondairement, ces forces interviennent dans le maintien de l'ordre public, la sécurisation des biens et permettent la libre circulation des biens et des personnes (ONU, 2008). Ainsi la mission des forces internationales pour le maintien de la paix n'implique pas la protection des ressources naturelles. Elles seraient donc restées passives devant la dégradation de la FCHS.

De 2006 à 2013, chacune des zones a subi une forte pression anthropique avec une quasi-disparition $\mathrm{du}$ couvert forestier. $\mathrm{La}$ disparition de la zone tampon en 2007 suite à la réunification des armés (ONUCI, 2007) n'a pas inversé la tendance à la déforestation de la FCHS. Désormais, le Nord qui bénéficiait jusque-là de la surveillance de la part des groupements rebelles est laissé pour compte donnant lieu à une disparition drastique de son couvert forestier pendant les conflits. Les mouvements des populations vers le Nord entre 2006 et 2013 en l'absence de surveillance seraient également dus à l'épuisement des terres au Centre et au Sud qui étaient sous pression anthropique depuis le début des conflits. D'autres aires protégées tel que le Mont Péko, situées en zone tampon ont subi une forte anthropisation du fait de l'absence des agents de surveillance (N'Goran, 2010). La différence d'administration n'implique aucune variation dans la dynamique des différentes zones de la FCHS.

\section{Approche méthodologique}

La classification supervisée des images Landsat de $30 \mathrm{~m}$ de résolution a permis d'établir des cartes d'occupation du sol et d'analyser la dynamique du paysage des zones Nord, Centre et Sud de la FCHS. L'évaluation de la précision de ces cartes a été faite par les méthodes généralement utilisées que sont la matrice de confusion et l'indice de Kappa (Pontius et al., 2001). L'indice de Kappa qui détermine dans la matrice de confusion la similitude entre les cartes et la réalité du terrain est compris entre 0 et 1 et peut être rangé en classe d'intervalle selon sa significativité. De 0 à 0,20 , il est très faible, de 0,21 à 0,40 il est faible, de 0,41 à 0,60 il modéré, 0,61 à 0,80 il est import et de 0,81 à 1 il est dit parfait (Chalifoux et al., 2006). Les indices de Kappa obtenus dans notre étude étant tous supérieurs à 0,81 nous pouvons dire que les cartes utilisées reflètent parfaitement les réalités du terrain.

La détermination des processus de changement du paysage se fait à travers la caractérisation de la structure paysagère. Pour ce faire plusieurs indices ont été proposés (Farina, 2000; Bogaert et Mahamane, 2005). Certains processus sont fréquemment observés pour des classes que pour d'autres. Par exemple la fragmentation est spécifique des milieux naturels telles que les forêts (Bogaert et al., 2011). En effet, la fragmentation entraine une réduction de l'aire moyenne et une augmentation du nombre de taches (Bogaert et al., 2002). Ainsi nous avons utilisé des indices qui émanent de la définition de la fragmentation qui sont l'aire moyenne et 
le nombre de taches (Bogaert et al 2002). Nous avons utilisé en plus de ces deux indices l'indice de dominance de la plus grande tache pour étayer la diminution de l'aire des taches (Bamba et al., 2010)

La fragmentation de la classe forestière résulte de sa conversion en culture et en habitation. La matrice dans les zones Nord, Centre et Sud de la FCHS a subi le même niveau de pression anthropique. Ces résultats confirment les travaux déjà effectués dans la FCHS et ceux de Hansen et al. (2008) et Chatelain (2015), qui ont montré une rapide conversion des terres du fait des conflits en Afrique au cours des dernières décennies.

Par l'analyse de la dynamique des différentes zones, cette étude met en évidence l'impartialité des forces internationales face à la destruction du couvert forestier ivoirien au cours de la période des conflits. Aussi l'étude met en exergue les dommages causés aux aires protégées par la population suite aux instabilités politiques comme ce fut le cas au Togo (Adjonou et al., 2010).

A travers la matrice de transition et l'indice de stabilité, on perçoit clairement l'évolution du couvert forestier vers les classes anthropiques et donc du risque de disparition des espèces primaires. En effet, la matrice de transition permet d'analyser les changements dans la composition du paysage tandis que l'indice de stabilité permet quant à lui de juger de la permanence du paysage initial (Bogaert et al., 2014).

\section{Conclusion}

Pour une conservation des ressources naturelles en période de conflits, l'Organisation des Nations Unies devrait adopter une loi sur le devoir de surveillance de ces ressources par les forces de maintien de la paix. Une autre solution pour pallier l'effondrement des ressources naturelles en période de conflits serait la mise en place d'une force entièrement dédiée à la surveillance de ces ressources.

Cette étude ne donne pas de preuves inconditionnées sur l'implication des différentes forces dans la gestion de la FCHS pendant les conflits. Une étude complémentaire serait une enquête sociologique auprès des populations infiltrées dans chacune des zones pour confirmer ou infirmer les résultats de cette étude.

\section{CONFLIT D'INTERETS}

Les auteurs déclarent ne pas avoir de conflit d'intérêts concernant cet article.

\section{CONTRIBUTIONS DES AUTEURS}

ATMK et YSSB sont les initiateurs du projet de recherche et de son exécution. Ils sont les auteurs de la rédaction de la méthodologie, de l'analyse et de l'interprétation des données. IB a contribué à l'analyse des données à travers la classification des images. SK, JYK et JB ont contribué à la révision critique du contenu de l'article.

\section{REFERENCES}

Adjonou K, Djiwa O, Kombate Y, Kokutse AD, Kokou K. 2010. Etude de la dynamique spatiale et structure des forêts denses sèches reliques du Togo: implications pour une gestion durable des aires protégées. Int. J. Biol. Chem. Sci., 4(1): 168-183.

Assalé AAY, Barima YSS, Kouakou KA, Kouakou ATM, Bogaert J. 2016. Agents de dégradation d'une aire protégée après une décennie de conflits en Côte d'Ivoire : cas de la forêt classée du HautSassandra. IJISR, 22(1): 123-133.

Ariori SL, Ozer P. 2005. Evolution des ressources forestières en Afrique de l'Ouest soudano-sahélienne au cours des 50 dernières années. Geo-Eco-Trop., 29: 61-68.

Arroyo-Rodriguez V, Pineda E, Escobar F, Benitez-Malvido J. 2009. Value of small Patches in the Conservation of PlantSpecies Diversity in Highly Fragmented Rainforest. Conserv. Biol., 23(3): 729739 . DOI: $\quad 10.1111 / \mathrm{j} .1523-$ 1739.2008.01120.x.

Bakayoko A, Martin P, Chatelain C, Traore D, Gautier L. 2011. Diversity, family dominance, life forms and ecological strategies of forest fragments compared to continuous forest in southwestern 
Côte d'Ivoire. Candollea, 66(2): 255262.

Bamba I. 2010. Anthropisation et dynamique spatiotemporelle de paysages forestiers en République démocratique du Congo. Thèse de doctorat, Université Libre de Bruxelles, Belgique, p.189.

Barima YSS, Kouakou ATM, Bamba I, Sangne YC, Godron M, Andrieu J, Bogaert J. 2016. Cocoa crops are destroying the forest reserves of the classified forest of Haut-Sassandra (Ivory Coast). Global Ecol Conserv., 8: 85-98.

Barima YSS, Barbier N, Ouattara B, Bogaert J. 2010. Relation entre la composition floristique et des indicateurs de la fragmentation du paysage dans une région de transition forêt-savane ivoirienne. Biotechnol. Agron. Soc. Environ., 14(4): 617-625. DOI : http://popups.ulg.ac.be/17804507/index.php?id $=6345$

Barima YSS, Barbier N, Bamba I, Traore D, Lejoly J, Bogaert J. 2009. Dynamique paysagère en milieu de transition forêtsavane ivoirienne. Bois For. Trop., 299(1): 15-25.

Bogaert J, Zhou L, Tucker CJ, Myneni RB, Ceulemans R. 2002. Evidence for a persistent and extensive greening trend in Eurasia inferred from satellite vegetation index data. J. Geophys. Res., 107(D11): 4119.4 DOI: 10.1029/2001JD001075.

Bogaert J, Ceulemans R, Salvador-Van ED. 2004. Decision tree algorithm for detection of spatial processes in landscape transformation. Environ. Manage., 33(1): 62-73.

Bogaert J, Mahamane A. 2005. Ecologie du paysage : cibler la configuration et l'échelle spatiale. Annales des Sciences Agronomiques du Bénin, 7(1): 39-68.

Bogaert J, Barima YSS, Ji J, Jiang H, Bamba I, Mongo LIW, Mama A, Nyssen E, Dahdouh-Guebas F, Koedam N. 2011. A Methodological Framework to Quantify Anthropogenic Effects on Landscape Patterns. In Landscape Ecology in Asian
Cultures, Hong S-K., Wu J, Kim J-E, Nakagoshi N (Eds). Springer: 141-167.

Bogaert J, Vranken I, Andre M. 2014. Anthropogenic effects in landscapes: historical context and spatial pattern. In Biocultural Landscapes Diversity, Functions and Values, Hong S-K, Bogaert J, Min Q (Eds). Springer Science + Business Media Dordrescht: 89-112.

Chander G, Markham BL. 2003. Revised Landsat-5 TM radiometric calibration procedures, and post-calibration dynamic ranges. IEEE Trans. Geosci. Remote Sens., 41(11): 2674-2677. DOI: 10.1109/TGRS.2003.818464

Chatelain C. 2015. Disparition de la forêt ivoirienne. Conservatoire et jardin botanique de la ville de Genève, p.16.

Chalifou XS, Nastev M, Lamontagne C, Latifovic R, Fernandes R. 2006. Cartographie de l'occupation et de l'utilisation du sol par imagerie satellitaire Landsat en hydrogéologie. Télédétection, 6(1): 9-17.

Dan CBS, Sinsin BA, Mensah GA, Lejoly J. 2012. Influence des activités anthropiques sur la diversité floristique des communautés végétales de la forêt marécageuse de Lokoli au Sud-Bénin. Int. J. Biol. Chem. Sci., 6(6): 3064-3081. http://dx.doi.org/10.4314/ijbcs.v6i6.8

Da Silva JMC, Tabarelli M. 2000. Tree species impoverishment and the future flora of the Atlantic forest of northeast Brazil. Nature, 404(6773): 72-74. DOI: $10.1038 / 35003563$

Debinski DM, Ray C, Saveraid EH. 2001. Species diversity and the scale of the landscape mosaic: do scales of movement and patch size affect diversity? Biol. Conserv., 98(2): 179190. http://dx.doi.org/10.1016/S00063207(00)00153-1

Dibloni OT, Coulibaly ND, Guenda W, Vermeulen C, Belem/Ouedraogo $\mathrm{M}$. 2009. Caractérisation paysanne de Hippopotamus amphibius Linné 1758, dans la Réserve de Biosphère de la Mare aux Hippopotames, en zone sud 
soudanienne du Burkina Faso. Int. J. Biol. Chem. Sci., 3(2): 386-397.

Farina A. 2000. Principles and Methodes in Landscape Ecology. Kluwers Academic Publishers: USA; p.235.

FAO. 2012. Situation des forêts du monde. Organisation des Nations Unies pour l'alimentation et l'agriculture Rome, 2012, p. 66.

Filho MS, Steiner A. 2005. Restauration des paysages forestiers. Série technique OIBT. $23: 1-34$.

Hansen MC, Stehman SV, Potapov PV, Loveland TR, Townshend JRG, Defries RS, Pittman KW, Arunarwati B, Stolle F, Steininger MK, Mark C, Dimiceli C. 2008. Humid Tropical Forest Clearing from 2000 to 2005 Quantified by Using Multitemporal and Multiresolution Remotely Sensed Data, PNAS. Nat. Aca. Sci., 105(27): 9439-9444.

Havyarimana F. 2015. La contribution de l'instabilité sociopolitique dans l'anthropisation des paysages au Burundi : dynamique spatiale et biodiversité. Thèse de doctorat, Université Libre de Bruxelles, Belgique, p. 293.

Koffie-Bikpo CY, Kra KS. 2013. La région du Haut-Sassandra dans la distribution des produits vivriers agricoles en Côte d'Ivoire. GEOTROPE, 2: 95-103.

kouakou ATM, Barima YSS, Kouakou KA, Kouame NF, Bogaert J, Kouadio YJ. 2015a. Forest dynamics in the north of the classified forest of Haut-Sassandra during the period of armed conflicts in Ivory Coast. American J. of L. Sci., 3(5): 375-382.

DOI: 10.11648/j.ajls.20150305.17

Kouakou KA, Barima YSS, Kouakou ATM, Sangne YC, Bamba I, Kouame NF. 2015b. Diversité végétale post-conflits armés de la forêt classée du HautSassandra (Centre-Ouest de la Côte d'Ivoire). J. Anim Plant Sci., 26(2): 4058-4071.

Kouame NF. 1998. Influence de l'exploitation forestière sur la végétation et la flore de la forêt classée du Haut-Sassandra (Centre-Ouest de la Côte d'Ivoire). Thèse de Doctorat de troisième cycle de
l'Université de Cocody (Abidjan), p. 203.

Martin P. 2008. Influence de la fragmentation forestière sur la régénération des espèces arborées dans le Sud-Ouest de la Côte d'Ivoire. Thèse de doctorat de l'Université de Genève, p. 306.

Mcgarigal K, Cushman SA. 2002. Comparative evaluation of experimental approaches to the study of habitat fragmentation effects. Ecological Applications, 12(2): 335-345.

N'da DH, Adou YCY, N'guessan KE, Kone M, Sagne YC. 2008. Analyse de la diversité floristique du parc national de la Marahoué, Centre-Ouest de la Côte d'Ivoire. Afrique Science, 4(3): 552-579.

N'goran RK. 2010. Application de l'évaluation environnementale stratégique dans un contexte conflictuel en Côte d'Ivoire. Essai présenté au Centre Universitaire de Formation en Environnement de l'université de Sherbrooke en vue de l'obtention du grade de Maître en environnement, p. 83.

OFAC. 2012. Les forêts du bassin du Congo État des forêts 2010. Office des publications de l'union européenne, Luxembourg, p. 276.

ONU. 2008. Opérations de maintien de la paix des Nations Unies. Principes et Orientations, p. 57.

ONUCI. 2007. Le contexte. https://onuci.unmissions.org/sites/default /files/old_spip (Site consulté le 26 février 2017).

Oszwald J, Lefebvre A, Arnauld X, Sartre D, Thalès M, Gond V. 2010. Analyse des directions de changement des états de surface végétaux pour renseigner la dynamique $\mathrm{du}$ front pionnier de Maçaranduba (para, brésil) entre 1997 et 2006. Télédétection, 9(2) : 97-111.

Oszwald J. 2005. Dynamique des formations agroforestières en Côte d'Ivoire (des années 1980 aux années 2000) suivi par télédétection et développement d'une approche cartographique. Thèse de doctorat de géographie. Université des sciences et technologies de Lille, p. 304. 
Oszwald J, Bigot S, Brou YT. 2003. Evolution géo-historique de la forêt classée du Haut-Sassandra (Côte d'Ivoire), p. 9.

PNUE. 2015. Evaluation environnementale post-conflit en Côte d'Ivoire, p. 160.

PNUE. 2011. Evaluation environnementale post-conflit en République démocratique du Congo. Synthèse à l'intention des décideurs, p. 76.

PNUE. 2006. Evaluation environnementale post-conflit du Soudan. Rapport de syntheses, p. 16.

Pontius JrRG, Cornell J, Hall C. 2001. Modeling the spatial pattern of land-use change with Geomod2: applica- tion and validation for Costa Rica. Agric Ecosyst Environ, 85(1-3): 191-203.

Sangne YC, Barima YSS, Bamba I, N'Doume, C-TA. 2015. Dynamique forestière post-conflits armés de la
Forêt classée du Haut-Sassandra (Côte d'Ivoire). Vertigo, 15(3): 1-18. DOI : 10.4000 /vertigo. 16784

Tente B, Baglo MA, Dossoumou JC, Yédomonhan H. 2011. Impacts des activités humaines sur les ressources forestières dans les terroirs villageois des communes de Glazoué et de DassaZoumè au centre-Bénin. Int. J. Biol. Chem. Sci., 5(5): 2022-2030. DOI : http://dx.doi.org/10.4314/ijbcs.v5i5.22

Zanh GG, Barima YSS, Kouakou KA, Sangne YC. 2016. Usages des produits forestiers non-ligneux selon les communautés riveraines de la forêt classée du HautSassandra (Centre-Ouest de la Côte d'Ivoire). Int. J. Pure App. Biosci. 4(5): 212-225.

DOI:

http://dx.doi.org/10.18782/2320-

7051.2346 